\title{
Kinematic Reliability Analysis of a 7-DOF Redundant Robot
}

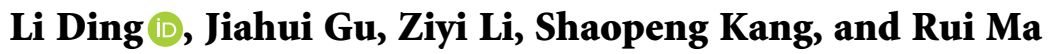 \\ College of Mechanical Engineering, Jiangsu University of Technology, Changzhou 213001, China \\ Correspondence should be addressed to Li Ding; nuaadli@163.com
}

Received 25 October 2021; Revised 11 January 2022; Accepted 20 January 2022; Published 28 February 2022

Academic Editor: Changsheng Li

Copyright $\odot 2022$ Li Ding et al. This is an open access article distributed under the Creative Commons Attribution License, which permits unrestricted use, distribution, and reproduction in any medium, provided the original work is properly cited.

\begin{abstract}
The kinematic reliability of robots, defined as the probability that the end-effector falls inside the specified safe boundary, is of great significance in predicting the accuracy achieved in reality. This work selects the 7 degrees-of-freedom (7-DOF) redundant robot as an example to conduct reliability analysis by utilizing the envelope method against time-related issues in this work. Since variables in industrial robots are very small relative to their means, the motion error functions are commonly linearized by the first-order Taylor's formula to simplify calculation, and the failure models in all directions and attitude angles are then established through the probability method over the entire input interval. As a result, the actual accuracy of the robot in each pose component will be displayed, instead of merely considering the position error like other scholars. The principle of the proposed method is to transform a time-dependent problem into a time-independent one with the help of the failure extreme points and endpoints, so as to enhance the operation efficiency under the premise of ensuring accuracy. Finally, the simulation results verify that the relative error of the envelope method is less than $6.0 \%$ compared with that of the Monte Carlo simulation method, and the computational efficiency is higher than that of the Monte Carlo method, which demonstrates that the envelope method has better comprehensive performance.
\end{abstract}

\section{Introduction}

Industrial robots are critical components of modern manufacturing because they offer low production costs, high stability, and high efficiency [1-3]. The 7-DOF redundant robot serves as a current research hotspot, famous for its tremendous flexibility in completing multiple tasks like avoiding singularity, avoiding obstacles, and optimizing pathways. Although the structure of multiaxis and multilink makes operations more convenient, they further magnify the uncertainty of the ontology, leading to the error accumulation problem becoming more prominent. Since errors universally exist in the manufacturing, assembly, and joint rotation processes [4,5], the actual pose of the end-effector may differ from the predetermined one where the precision is in perfect condition. Therefore, kinematic reliability, which is widely used in mechanical structures, is proposed as a method of uncertainty evaluation to quantify the impact of multiple factors on accuracy.

For the inverse kinematics of 7-DOF redundant robots, studies have shown that some algorithms can make the pose of the manipulator almost identical to the ideal state. Dereli and Köker [6] employed a group optimization technique called the firefly algorithm to narrow the error between the end-effector and the target point to $10^{-10} \mathrm{~mm}$. This did not, however, imply that robots will be able to achieve such precision in practical engineering owing to the influence of external factors that cannot be eliminated. In general, the link error of industrial robots ranges from $0.01 \mathrm{~mm}$ to $1 \mathrm{~mm}$ $[7,8]$. On the other hand, the rotation error, as well as other errors, cannot be overlooked. These variables, in contrast to the inverse kinematics algorithm, significantly disrupt the working condition of the manipulator. Furthermore, intelligent welding work performed by robots in conjunction with vision systems has been progressing apace in recent years [9], whose kinematic error can be effectively predicted based on the reliability theory. All of this suggests that kinematic robot reliability is an essential study area with several applications.

The kinematic reliability problem of robots can be divided into two categories: point reliability and time-dependent reliability [10-13]. The instantaneous failure rate at 
a point on the trajectory is reflected in point reliability regardless of whether motion is valid at other points, which commonly occurs during the measurement of the repeatability of positioning. Due to the low complexity and frequent occurrences of this problem, it has become the primary research type for scholars. The first-order reliability method (FORM) and the Monte Carlo simulation (MCS) method are two typically used calculation methods $[14,15]$, with the latter serving as a benchmark for the new method in that it has higher accuracy based on a large number of sample simulations but higher calculation cost [16]. In addition, Zhang and Xu [17] used a combination of saddle point approximation (SPA) and dimension reduction (DR) to forecast the precision of a 6-DOF industrial robot, filling a gap in the nonlinear error literature. However, in engineering practice, the reliability of continuous trajectory is of higher application value since it can reveal the laws of robot terminal motion in global terms, which is why the timedependent problem of kinematic reliability receives more attention.

To be more specific, it refers to the likelihood that an arbitrary discrete point on its terminal trajectory falls within a presupposed tolerance zone, the center of which is on the desired path [18-20]. Nevertheless, the true motion accuracy is difficult to estimate on account of the high correlation between discrete points at different times and the accumulation of failure rates over time. Regrettably, almost all time-dependent problem research objects are four-bar mechanisms with a single output and few variables in the last decade, where popular algorithms such as Rice's formula, extreme value method, and envelope method [21-23] have been successfully implemented.

With the improvement of relevant theories over time, some algorithms are appropriately used for robot timedependent reliability analysis after reasonable modification. Zhao et al. [24] introduced the first-passage method into the analysis of the 6-DOF robot's pose error, showing that the method's accuracy is fairly good when accounting for low relationships between discrete points, but the calculation area is limited to situations with a very low failure rate. Pendey and Zhang [25] effectively computed the fluctuation range around the intended motion, and the probability density function of the extreme value distribution points is derived in terms of the principle of maximum entropy. Whereas this method requires a large amount of sample data, its stability may be insufficient for widespread adoption. In order to solve the problem that the failure probability of the $3 \mathrm{R}$ planar robot is difficult to estimate, Vieira et al. [26] coupled the interval analysis method with the MCS method, taking advantage of both to formulate the law of plane motion, but it cannot guarantee its adaptability to spatial motion. Given the considerable limitations of the method mentioned above in the investigation of time-dependent reliability, a new method is indispensable to assess the robot from various perspectives.

Motivated by the abovementioned studies and the huge application potential of kinematic reliability, this article chooses the 7-DOF redundant robot as the research object. The main contributions are threefold: (1) compared with planar mechanisms or 6-DOF manipulators, the 7-DOF redundant robot involves more input variables and higher coupling in motion function, whose time-dependent reliability problem has not been studied yet. (2) The simulation analysis in this article, conducted by the enveloped method, including three positions and three attitude angles, is remarkably distinct from those that just focus on position errors to better display the components of the total error. (3) The error function of the comprehensive pose obeying nonlinear is approximated on the basis of the multidimensional normal integral.

The framework of this article is as follows. In Section 2, we elaborate on the robotic kinematics. Section 3 deduces error functions and kinematic reliability models. We propose the envelope method in Section 4. A simulation example is presented in Section 5, and the conclusions are summarized in Section 6.

\section{Robot Kinematics}

To begin with, the forward kinematics of the robot should be established to construct the error function, which describes the transfer relationship between connecting rod movements. A classic method, the improved DH parameter [27], identifies the internal relativity between two neighboring links through four parameters in the definite coordinate systems, as shown in Figure 1 . The symbols $a_{i-1}, \alpha_{i-1}, d_{i}$, and $\theta_{i}$ of four parameters represent link length, link twist, link offset, and joint angle, respectively, listed in Table 1.

The homogeneous transformation matrix ${ }_{i}^{i-1} T$ of adjacent frames is described as

$$
{ }_{i}^{i-1} T=\left[\begin{array}{cccc}
c \theta_{i} & -s \theta_{i} & 0 & a_{i-1} \\
s \theta_{i} c \alpha_{i-1} & c \theta_{i} c \alpha_{i-1} & -s \alpha_{i-1} & -s \alpha_{i-1} d_{i} \\
s \theta_{i} s \alpha_{i-1} & c \theta_{i} s \alpha_{i-1} & c \alpha_{i-1} & c \alpha_{i-1} d_{i} \\
0 & 0 & 0 & 1
\end{array}\right],
$$

where $c$ is the abbreviation of the symbol cos and $s$ is the abbreviation of the symbol sin.

According to the progressive relationship between the connecting rods, the pose matrix ${ }_{i}^{0} T$ of end-effector is given by

$$
{ }_{i}^{0} T={ }_{1}^{0} T \cdot{ }_{2}^{1} T \cdots \cdot{ }_{i}^{i-1} T=\left[\begin{array}{cccc}
n_{x} & o_{x} & a_{x} & p_{x} \\
n_{y} & o_{y} & a_{y} & p_{y} \\
n_{z} & o_{z} & a_{z} & p_{z} \\
0 & 0 & 0 & 1
\end{array}\right],
$$

where $\quad n=\left[n_{x}, n_{y}, n_{z}\right]^{T}, \quad o=\left[o_{x}, o_{y}, o_{z}\right]^{T}, \quad$ and $a=\left[a_{x}, a_{y}, a_{z}\right]^{T}$ are all unit vector projected onto the fixed coordinate frame. $p=\left[p_{x}, p_{y}, p_{z}\right]^{T}$ is a position vector in the fixed coordinate frame.

\section{Kinematic Reliability Model}

The kinematic reliability of a robot can be defined as the probability that the actual output trajectory of its end-effector in the specified interval falls within the allowable error 


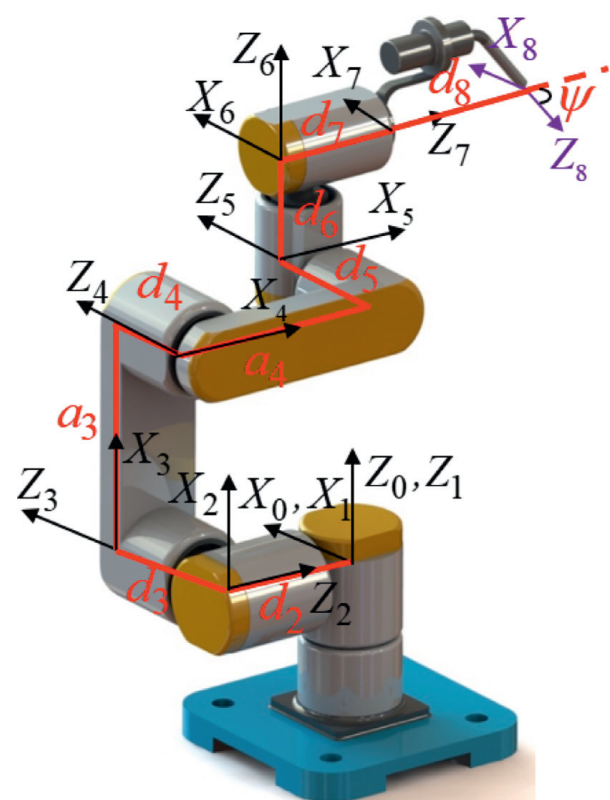

FIgURE 1: Schematic diagram of the 7-DOF redundant robot.

TABLE 1: Improved D-H parameters of the 7-DOF redundant robot.

\begin{tabular}{ccccc}
\hline$i$ & $a_{i-1}(\mathrm{~mm})$ & $\alpha_{i-1}\left({ }^{\circ}\right)$ & $d_{i}(\mathrm{~mm})$ & $\theta_{i}\left({ }^{\circ}\right)$ \\
\hline 1 & 0 & 0 & 0 & $\theta_{1}$ \\
2 & 0 & 90 & -297.5 & $\theta_{2}$ \\
3 & 0 & 90 & 355.5 & $\theta_{3}$ \\
4 & 450 & 0 & -293 & $\theta_{4}$ \\
5 & 400 & 0 & 255 & $\theta_{5}$ \\
6 & 0 & 90 & 197 & $\theta_{6}$ \\
7 & 0 & 90 & 104 & $\theta_{7}$ \\
\hline
\end{tabular}

range. In order to vividly describe the influence of uncertain factors on the accuracy of the trajectory, Figure 2 is used to depict the difference between the failed trajectory (red curve), the qualified trajectory (black curve), and the ideal trajectory (purple curve).

In the past, it has been discovered that the error of a robot is primarily caused by the geometric dimension of the connecting rod and the joint rotation [8]. Furthermore, link offset should be considered due to inevitable deviation from assembly and joint wear, which is easily overlooked. In engineering practice, however, the standard deviation of each variable is controlled within a small range, usually less than one-thousandth relative to the corresponding mean value. Based on the research results from the literature $[11,17,28]$, the error distribution of each variable is assumed to be normal and mutually independent. Then, the error function $g_{j}(\mathbf{X}, \theta)(j=1,2, \cdots, 6)$ of end-effector in three positions and three attitude angles, is described as

$$
g_{j}(\mathbf{X}, \boldsymbol{\theta})=\psi_{j}(\mathbf{X}, \boldsymbol{\theta})-\psi_{j}(\boldsymbol{\theta})
$$

where $\psi_{j}(\mathbf{X}, \theta)$ and $\psi_{j}(\theta)$ are the actual output value and theoretical output value, respectively, and $n$ is the dimension of the vector $\mathbf{X}=\left[X_{1} \cdots X_{n}\right]^{T}$.

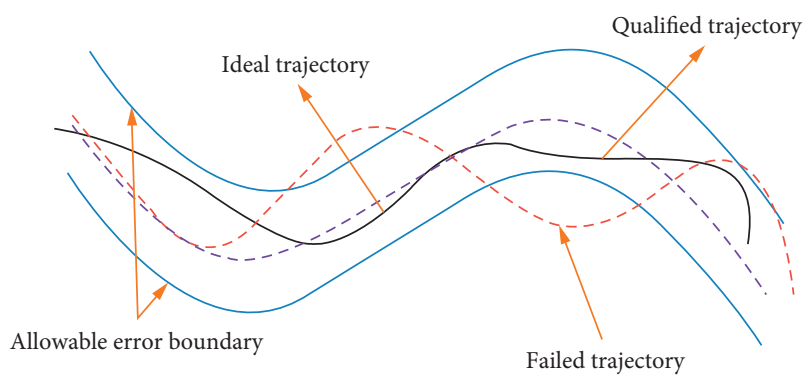

FIGURE 2: Difference of three trajectories.

When the magnitude of standard deviation of the random variable is much smaller than the counterpart of the mean value, the first-order Taylor formula [29] is an effective treatment for approximating the error function. As a result, the error function can be expanded at the mean value of $\mathbf{X}$, written as

$$
g_{j}(\mathbf{X}, \boldsymbol{\theta}) \approx a_{j 0}(\boldsymbol{\theta})+a_{j}(\boldsymbol{\theta}) \cdot(\boldsymbol{\mu}-\mathbf{X}),
$$

where $a_{j 0}=\psi_{j}(\mu, \theta)-\psi_{j}(\theta), a_{j}(\theta)=\left(\partial \psi_{j} /\left.\partial X_{i}\right|_{\mu}\right)_{n \times 1}$.

Remark 1. In this article, all variables follow normal distribution, so the correlation property can be used to further simplify the operation.

Property 1. If $X \sim N\left(\mu, \sigma^{2}\right)$, there is

$$
a X+b \sim N\left(a \mu+b,(a \sigma)^{2}\right)
$$

where $a$ and $b$ are both real numbers.

Therefore, $\mathbf{X}$ subject to the normal distribution should be transformed into $\mathbf{U}$ subject to the standard normal distribution, given by

$$
U_{i}=\frac{X_{i}-\mu_{i}}{\sigma_{i}} .
$$

So equation (3) can be rewritten as

$$
g_{j}(\mathbf{U}, \boldsymbol{\theta}) \approx L_{j}(\mathbf{U}, \boldsymbol{\theta})=b_{j 0}(\boldsymbol{\theta})+b_{j}(\boldsymbol{\theta}) \cdot \mathbf{U},
$$

where $b_{j 0}(\theta)=a_{j 0}(\theta), b_{j}(\theta)=\left(a_{j i}(\theta) \sigma_{i}\right)_{n \times 1}, a_{j i}$ is the $i$-th element of $a_{j}$, and $\sigma_{i}$ is the standard deviation of $X_{i}$.

Since the joint angle changes with time $t, g_{j}(\mathbf{U}, \theta)$ can be converted into a time-dependent function. If moving time $t$ is set in the interval $\left[t_{o}, t_{e}\right]$, taking the position $x$ of the endeffector as an example, its kinematic reliability model can be defined as

$$
R_{1}\left(t_{o}, t_{e}\right)=\operatorname{Pr}\left\{\left|g_{1}(\mathbf{X}, t)\right| \leq \varepsilon, \forall t \in\left[t_{o}, t_{e}\right]\right\} .
$$

The corresponding failure probability model can be expressed as

$$
\begin{aligned}
p_{f 1}\left(t_{o}, t_{e}\right) & =1-R_{1}\left(t_{o}, t_{e}\right) \\
& =\operatorname{Pr}\left\{\left|g_{1}(\mathbf{X}, t)\right|>\varepsilon, \forall t \in\left[t_{o}, t_{e}\right]\right\} .
\end{aligned}
$$

Similarly, the comprehensive reliability model of the robot end-effector is 


$$
R\left(t_{o}, t_{e}\right)=\operatorname{Pr}\left\{\bigcap_{j=1}^{6}\left|g_{j}(\mathbf{X}, t)\right| \leq \varepsilon, \forall t \in\left[t_{o}, t_{e}\right]\right\} .
$$

The comprehensive pose failure model of the robot endeffector is

$$
p_{f}\left(t_{o}, t_{e}\right)=\operatorname{Pr}\left\{\bigcup_{j=1}^{6}\left|g_{j}(\mathbf{X}, t)\right|>\varepsilon, \forall t \in\left[t_{o}, t_{e}\right]\right\} .
$$

According to the preceding derivation, if a trajectory is eligible in interval $\left[t_{o}, t_{e}\right]$, the error of a point at any time must satisfy the condition that the error is within the tolerance sphere. Nonetheless, calculating the error at each point in reality is unaffordable. As a result, it is critical to develop a high-precision and high-efficiency algorithm to replace the MCS method for solving the kinematic reliability problem.

\section{Envelope Method}

The linchpin of the envelope method is to utilize failure boundary functions $G_{j}^{+}(\mathbf{X})=0$ and $G_{j}^{-}(\mathbf{X})=0$ converting reliability problem into a time-independent problem. $G_{j}^{+}(\mathbf{X})=0$ and $G_{j}^{-}(\mathbf{X})=0$ are limit state functions, which are tangent to hypersurfaces $g_{j}(\mathbf{X}, t)=\varepsilon$ and $g_{j}(\mathbf{X}, t)=-\varepsilon$, respectively.

According to this nature, there is

$$
\frac{g_{j}(\mathbf{X}, t+\Delta t)-g_{j}(\mathbf{X}, t)}{\Delta t}=0 \text {. }
$$

If $\Delta t \longrightarrow 0$, we have

$$
\frac{\partial g_{j}(\mathbf{X}, t)}{\partial t}=0 \text { or } \dot{g}_{j}(\mathbf{X}, t)=0 .
$$

Then, $G_{j}^{+}(\mathbf{X})$ is expressed as follows:

$$
\left\{\begin{array}{l}
g_{j}(\mathbf{X}, t)=\varepsilon \\
\dot{g}_{j}(\mathbf{X}, t)=0
\end{array}\right.
$$

We solve equation (14) to obtain

$$
G_{j}^{+}(\mathbf{X})=g\left(X, \dot{g}_{j}^{-1}(\mathbf{X})\right)-\varepsilon=0,
$$

where $\dot{g}_{j}^{-1}(\cdot)$ is the inverse function of $\dot{g}_{j}(\cdot)$ with respect to $t$.

So are $G_{j}^{-}(\mathbf{X})$ as

$$
\left\{\begin{array}{l}
g_{j}(\mathbf{X}, t)=-\varepsilon \\
\dot{g}_{j}(\mathbf{X}, t)=0
\end{array}\right.
$$

There are also

$$
G_{j}^{-}(\mathbf{X})=g\left(X, \dot{g}_{j}^{-1}(\mathbf{X})\right)+\varepsilon=0 .
$$

After the above derivation about $G_{j}^{+}(\mathbf{X})$ and $G_{j}^{-}(\mathbf{X})$, the kinematic reliability model of the robot becomes

$$
R_{j}\left(t_{o}, t_{e}\right)=\operatorname{Pr}\left\{G_{j}^{+}(\mathbf{X})<0 \cap G_{j}^{-}(\mathbf{X})>0, \forall t \in\left[t_{o}, t_{e}\right]\right\} .
$$

Since it is very difficult to obtain the expression of the inverse function $\dot{g}_{j}^{-1}(\mathbf{X})$ directly, $G_{j}^{+}(\mathbf{X})$ and $G_{j}^{-}(\mathbf{X})$ need to be simplified further.
Substituting equation (7) into equation (14), $G_{j}^{+}(\mathbf{X})$ is rewritten as

$$
\left\{\begin{array}{l}
L_{j}=b_{j 0}(t)+\mathbf{b}_{\mathbf{j}}(t) \cdot \mathbf{U}=\varepsilon, \\
\dot{L}_{j}=\dot{b}_{j 0}(t)+\dot{b}_{\mathbf{j}}(t) \cdot \mathbf{U}=0,
\end{array}\right.
$$

where $L_{j}$ is the abbreviation of $L_{j}(\mathbf{U}, t)$.

Referring to the research results of literature [30], the gradient of $L_{j}\left(\mathbf{U}, t_{i}^{+}\right)=\varepsilon$ and $\mathbf{U}$ are collinear at some instants, as shown in Figure 3.

Hence, $\mathbf{U}$ is equal to

$$
\mathbf{U}=c \frac{\mathbf{b}_{\mathbf{j}}(t)}{\sqrt{\mathbf{b}_{\mathbf{j}}(t) \cdot \mathbf{b}_{\mathbf{j}}(t)}},
$$

where $c$ is a constant.

We plug this into equation (19) to yield

$$
b_{j 0}(t)+c \frac{\mathbf{b}_{\mathbf{j}}(t) \cdot \mathbf{b}_{\mathbf{j}}(t)}{\sqrt{\mathbf{b}_{\mathbf{j}}(t) \cdot \mathbf{b}_{\mathbf{j}}(t)}}=\varepsilon .
$$

Therefore, $c$ is given by

$$
c=\frac{\left[\varepsilon-b_{j 0}(t)\right]}{\sqrt{\mathbf{b}_{\mathbf{j}}(t) \cdot \mathbf{b}_{\mathbf{j}}(t)}}
$$

Equation (19) can be transformed into

$$
\dot{b}_{j 0}(t)+\left[\varepsilon-b_{j 0}(t)\right] \frac{\dot{b}_{\mathbf{j}}(t) \cdot \mathbf{b}_{\mathbf{j}}(t)}{\mathbf{b}_{\mathbf{j}}(t) \cdot \mathbf{b}_{\mathbf{j}}(t)}=0 .
$$

On the basis of equation (23), the value of $t_{i}^{+}$can be solved. On the other hand, the number of solutions $t_{i}^{+}$may not be fixed due to different intricacies of trajectories. Ultimately, expansion point $\mathbf{U}\left(t_{i}^{+}\right)$is represented by

$$
\mathbf{U}\left(t_{i}^{+}\right)=\frac{\left[\varepsilon-b_{j 0}\left(t_{i}^{+}\right)\right] \mathbf{b}_{\mathbf{j}}\left(t_{i}^{+}\right)}{\mathbf{b}_{\mathbf{j}}\left(t_{i}^{+}\right) \cdot \mathbf{b}_{\mathbf{j}}\left(t_{i}^{+}\right)} .
$$

In the same way, $t_{i}^{-}\left(i=1,2 \cdots k^{-}\right)$can be obtained in line with $G_{j}^{-}(U)=0$ whose expression is

$$
\left\{\begin{array}{l}
L_{j}=b_{j 0}(t)+\mathbf{b}_{\mathbf{j}}(t) \cdot \mathbf{U}=-\varepsilon, \\
\dot{L}_{j}=\dot{b}_{j 0}(t)+\dot{b}_{\mathbf{j}}(t) \cdot \mathbf{U}=0 .
\end{array}\right.
$$

And expansion point $\mathbf{U}\left(t_{i}^{-}\right)$is described as the following expression:

$$
\mathbf{U}\left(t_{i}^{-}\right)=-\frac{\left[\varepsilon+b_{j 0}\left(t_{i}^{-}\right)\right] \mathbf{b}_{\mathbf{j}}\left(t_{i}^{-}\right)}{\mathbf{b}_{\mathbf{j}}\left(t_{i}^{-}\right) \cdot \mathbf{b}_{\mathbf{j}}\left(t_{i}^{-}\right)},
$$

where $t_{i}^{-}\left(i=1,2 \cdots k^{-}\right)$is the solution of equation (25).

Remark 2. Directly solving equations (19) and (25), both of which include about a thousand trigonometric functions, is difficult and takes an inordinate amount of time. An efficient way is to take points at appropriate intervals to find the closest solution among all samples. 


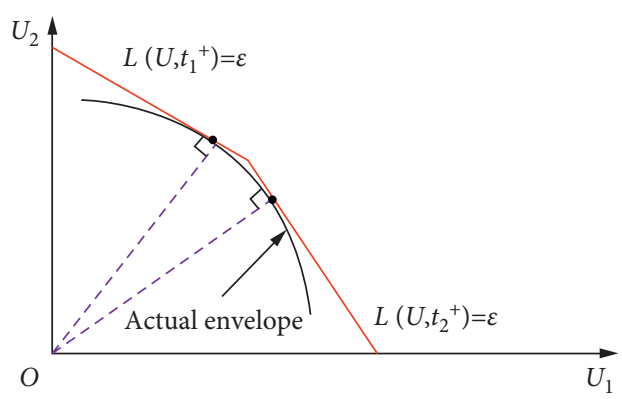

Figure 3: Intersections at instant time $t_{i}^{+}$.

Since the above derivation is reasonable, we use $L_{j}\left(\mathbf{U}, t_{i}^{+}\right)=\varepsilon$ and $L_{j}\left(\mathbf{U}, t_{i}^{-}\right)=-\varepsilon$ instead of $G_{j}^{+}(\mathbf{U})=0$ and
$G_{j}^{-}(\mathbf{U})=0$ to simplify the kinematic reliability model. There is

$$
R_{j}\left(t_{o}, t_{e}\right)=\operatorname{Pr}\left\{\left[\bigcap_{i}^{k^{+}} L_{j}\left(\mathbf{U}, t_{i}^{+}\right)<\varepsilon\right] \cap\left[\bigcap_{i}^{k^{-}} L_{j}\left(\mathbf{U}, t_{i}^{-}\right)>-\varepsilon\right], \forall t \in\left[t_{o}, t_{e}\right]\right\} .
$$

Beside time instant $t_{i}^{+}$and $t_{i}^{-}$, start time $t_{o}$ and end time $t_{e}$ should be also added to improve calculation accuracy. Consequently, we recast the kinematic reliability model as

$$
R_{j}\left(t_{o}, t_{e}\right)=\operatorname{Pr}\left\{\bigcap_{i}^{k} s\left(t_{i}\right) L_{j}\left(\mathbf{U}, t_{i}\right)<\varepsilon_{i}\right\},
$$

where $t_{i}$ contains $t_{o}, t_{e}, t_{i}^{+}, t_{i}^{-}$, and $k=k^{+}+k^{-}+2 . s\left(t_{i}\right)$ is the sign function of $L_{j}\left(\mathbf{U}, t_{i}\right)$, defined as

$$
s\left(t_{i}\right)= \begin{cases}+1, & \text { if } t_{i}=t^{+}, \\ -1, & \text { if } t_{i}=t^{-}, \\ +1, & \text { if } t_{i}=t_{0} \text { or } t_{e}, L\left(\mathbf{U}, t_{i}\right) \geq 0, \\ -1, & \text { if } t_{i}=t_{0} \text { or } t_{e}, L\left(\mathbf{U}, t_{i}\right)<0 .\end{cases}
$$

Combining the previous descriptions, $L(\mathbf{U}, t)$ is similar to $g_{j}(\mathbf{U}, t)$, which leads to the error distribution of $L(\mathbf{U}, t)$ being normal. In light of this property, the mean $\mu_{\mathrm{L}}$ and covariance matrix $\Sigma$ can be derived to compute the reliability $R\left(t_{o}, t_{e}\right)$, whose formula is

$$
R\left(t_{o}, t_{e}\right)=\Phi\left(\varepsilon, \boldsymbol{\mu}_{\mathrm{L}}, \boldsymbol{\Sigma}\right),
$$

where $\Phi(\cdot)$ is the cumulative distribution function (CDF) of multiple standard normal variables.

The formula of $\mu_{\mathrm{L}}$ is

$$
\boldsymbol{\mu}_{\mathrm{L}}=\left(s\left(t_{i}\right) \mu_{\mathrm{L}}\left(t_{i}\right)\right)_{i=1 \times k}=\left(s\left(t_{i}\right) b_{j o}\left(t_{i}\right)\right)_{i=1 \times k^{\prime}}
$$

where $\Sigma$ denotes the covariance matrix with $k^{2}$ elements, calculated by

$$
\sigma_{i j}=s\left(t_{i}\right) s\left(t_{j}\right) b\left(t_{i}\right) b\left(t_{j}\right) .
$$

Remark 3. It is worth noting that $\Sigma$ must be a positive definite matrix during the calculation process. This is to say, the $\Sigma$ is full-rank with a positive determinant value.
Otherwise, we must eliminate the point with the lowest failure rate one by one until the covariance matrix $\Sigma$ meets the above condition. The calculation formula for failure probability is written as

$$
\begin{aligned}
p_{j f}\left(t_{i}\right) & =\operatorname{Pr}\left\{s\left(t_{i}\right) L\left(U, t_{i}\right)>\varepsilon_{i}\right\} \\
& =2-2 * \Phi\left\{\frac{s\left(t_{i}\right)\left[\varepsilon-b_{j 0}\left(t_{i}\right)\right]}{\sqrt{b_{j}\left(t_{i}\right) \cdot b_{j}\left(t_{i}\right)}}\right\} .
\end{aligned}
$$

Immediately after this process, the $r$ effective points are kept to reconstitute the new mean $\mu_{\mathrm{L}}^{\prime}$ and matrix $\Sigma^{\prime}$, namely,

$$
\begin{aligned}
& \boldsymbol{\mu}_{\mathbf{L}}^{\prime}==\left(s\left(t_{i}^{\prime}\right) b_{j o}\left(t_{i}^{\prime}\right)\right)_{i=1 \times r}, \\
& \boldsymbol{\Sigma}^{\prime}=\left(\sigma_{i j}^{\prime}\right)_{r \times r},
\end{aligned}
$$

where $\sigma_{i j}^{\prime}=s\left(t_{i}^{\prime}\right) s\left(t_{j}^{\prime}\right) b\left(t_{i}^{\prime}\right) b\left(t_{j}^{\prime}\right)$.

Accordingly, the reliability of the robot end-effector is calculated by

$$
\begin{array}{r}
R\left(t_{o}, t_{e}\right)=\Phi\left(\varepsilon, \boldsymbol{\mu}_{\mathbf{L}}^{\prime}, \mathbf{\Sigma}^{\prime}\right) \\
=\int_{-\varepsilon}^{\varepsilon} \frac{1}{(2 \pi)^{r / 2}\left|\mathbf{\Sigma}^{\prime}\right|} \exp \left[-\frac{1}{2}\left(\mathbf{x}-\boldsymbol{\mu}_{\mathbf{L}}^{\prime}\right) \Sigma^{\prime}\left(\mathbf{x}-\boldsymbol{\mu}_{\mathbf{L}}^{\prime}\right)^{T}\right] \mathrm{d} x .
\end{array}
$$

Finally, we summarize the flow of kinematic reliability, as shown in Figure 4.

When the reliability values for the specified tolerances are obtained, the performance of the robot can be evaluated according to the quality indicator of the industry. In general, product accuracy must meet at least Three-Sigma (reliability $\geq 99.7 \%$ ) requirement for enterprises, but such a production situation is not clearly competitive due to a certain amount of profit applied to after-sales links and the low loyalty of customers. Therefore, the Six Sigma principle [31] (reliability $\geq 99.99966 \%$ ) has gradually become a new quality measure 


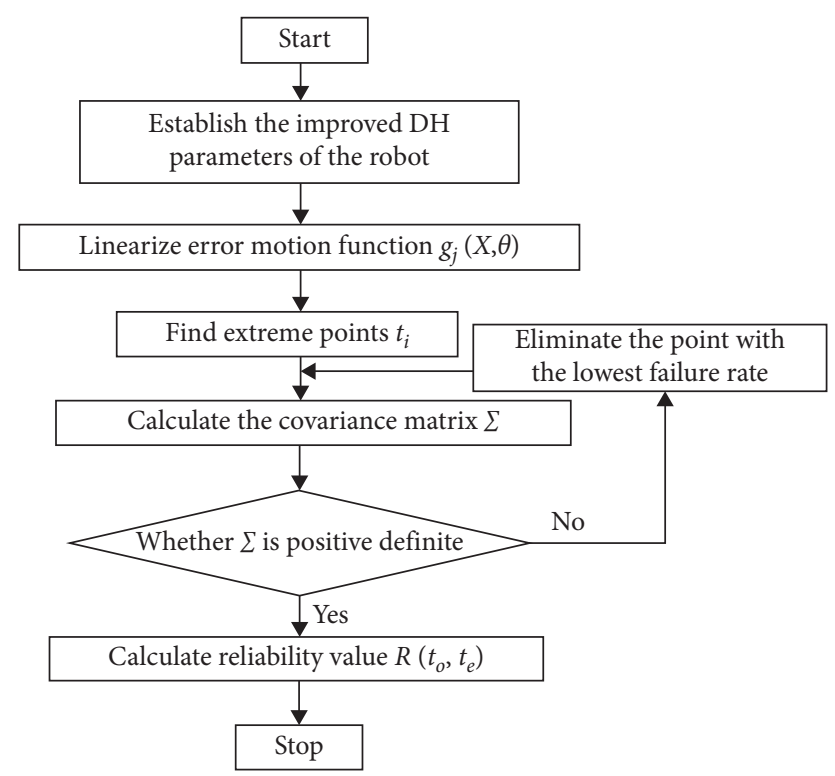

FIGURE 4: Flowchart of the envelope method.

since it was proposed in the 1990s. If the precision of the robot can meet such situation, it means that the product is almost impossible to defect.

\section{Simulation Results}

In this article, the simulation platform MATLAB 2019a is employed, and the CPU is an AMD Ryzen 7 3700X 8-Core. For example, to replicate the shipyard welding task, a 7-DOF redundant robot is chosen, with Table 2 displaying the corresponding variable distribution. Based on the robot kinematics literature, the cyclic coordinate descent (CCD) method [32] is used to calculate the corresponding joint angles of the robot, and we have $\theta_{o}=\left[-58^{\circ}, 45^{\circ},-7^{\circ}\right.$, $\left.48^{\circ}, 6^{\circ},-46^{\circ}, 37^{\circ}\right]$ and $\theta_{e}=\left[-31^{\circ}, 121^{\circ}, 69^{\circ}, 90^{\circ}, 85^{\circ}, 27^{\circ},-8\right]$, which are the starting angle and the ending angle, respectively. Furthermore, the following motion function of the robot joint angles within $3 \mathrm{~s}$ is planned:

$$
\theta_{j}(t)=\theta_{j}(0)+\frac{\theta_{j}(0)-\theta_{j}(0)}{T}\left[t-\frac{T}{2 \pi} \sin \left(\frac{2 \pi}{T} t\right)\right],
$$

where $\theta_{j}(\cdot)(j=1,2, \cdots, 7)$ represents the value of the joint angle $\theta_{j}$ and $T=3$ represents the total time of executing the task.

Figure 5 depicts the motion trajectory of the robot endeffector in the Cartesian coordinate system from the starting point to the ending point.

In order to demonstrate the validity of the envelope method, the equivalent extreme value (EEV) method [33] and MCS are introduced for comparison, with the latter serving as a desired value benchmark with $1 \times 10^{6}$ random trajectories simulated. For the MCS method, only 31 points on a trajectory with a $0.1 \mathrm{~s}$ interval are taken into account due to unaffordable computation of points at each time. If all of these points are within the allowable tolerance, this
TABLE 2: Mean and standard deviation of variables.

\begin{tabular}{lccc}
\hline Variables & Mean & Standard deviation & Distribution \\
\hline$x_{1}=a_{3}$ & $\mu_{1}=0.45 \mathrm{~mm}$ & $\sigma_{1}=2.9 \times 10^{-4} \mathrm{~mm}$ & Normal \\
$x_{2}=a_{4}$ & $\mu_{2}=0.4 \mathrm{~mm}$ & $\sigma_{2}=2.8 \times 10^{-4} \mathrm{~mm}$ & Normal \\
$x_{3}=d_{2}$ & $\mu_{3}=-0.2975 \mathrm{~mm}$ & $\sigma_{3}=1.6 \times 10^{-4} \mathrm{~mm}$ & Normal \\
$x_{4}=d_{3}$ & $\mu_{4}=0.3555 \mathrm{~mm}$ & $\sigma_{4}=2.4 \times 10^{-4} \mathrm{~mm}$ & Normal \\
$x_{5}=d_{4}$ & $\mu_{5}=-0.293 \mathrm{~mm}$ & $\sigma_{5}=2.2 \times 10^{-4} \mathrm{~mm}$ & Normal \\
$x_{6}=d_{5}$ & $\mu_{6}=0.255 \mathrm{~mm}$ & $\sigma_{6}=1.7 \times 10^{-4} \mathrm{~mm}$ & Normal \\
$x_{7}=d_{6}$ & $\mu_{7}=0.197 \mathrm{~mm}$ & $\sigma_{7}=1.9 \times 10^{-4} \mathrm{~mm}$ & Normal \\
$x_{8}=d_{7}$ & $\mu_{8}=0.104 \mathrm{~mm}$ & $\sigma_{8}=1.4 \times 10^{-4} \mathrm{~mm}$ & Normal \\
$x_{9}=d_{8}$ & $\mu_{9}=0.15 \mathrm{~mm}$ & $\sigma_{9}=1.9 \times 10^{-4} \mathrm{~mm}$ & Normal \\
$x_{10}=\theta_{1}$ & $\mu_{10}=\theta_{1}$ & $\sigma_{10}=4.16 \times 10^{-4}$ o & Normal \\
$x_{11}=\theta_{2}$ & $\mu_{11}=\theta_{2}$ & $\sigma_{11}=3.64 \times 10^{-4}$ o & Normal \\
$x_{12}=\theta_{3}$ & $\mu_{12}=\theta_{3}$ & $\sigma_{12}=1.34 \times 10^{-3}$ 。 & Normal \\
$x_{13}=\theta_{4}$ & $\mu_{13}=\theta_{4}$ & $\sigma_{13}=1.13 \times 10^{-3}$ 。 & Normal \\
$x_{14}=\theta_{5}$ & $\mu_{14}=\theta_{5}$ & $\sigma_{14}=9.4 \times 10^{-4}$ 。 & Normal \\
$x_{15}=\theta_{6}$ & $\mu_{15}=\theta_{6}$ & $\sigma_{15}=1.1 \times 10^{-3}$ 。 & Normal \\
$x_{16}=\theta_{7}$ & $\mu_{16}=\theta_{7}$ & $\sigma_{16}=1.42 \times 10^{-3}$ & Normal \\
\hline
\end{tabular}

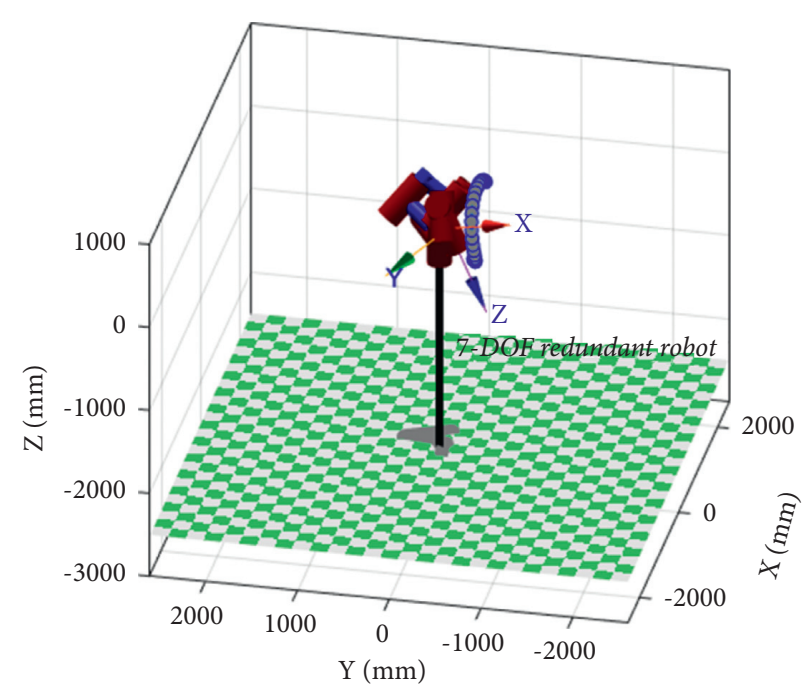

FIgURE 5: Motion trajectory of the end-effector.

trajectory is regarded as valid. Otherwise, this course is unqualified. When $t$ is at $3 \mathrm{~s}$, for example, failure samples and valid samples are displayed, as illustrated in Figure 6, with the comprehensive position error $\left(\sqrt{\sum_{i=1}^{3} g_{i}^{2}}\right)$ of representative trajectories shown in Figure 7.

Figure 8 plots the results of three algorithms. It can be seen that the failure rate of three algorithms decreases with the increment of error. From a global perspective, however, the envelope method has higher agreement with the MCS method. Since the EEV is commonly applied in conditions where there is an extreme point, it is not suitable for the case of small fluctuations in trajectory. This is why the accuracy of EEV is unstable for kinematic reliability.

According to the trajectory given above, taking the $y$ direction as an example, the time $t$ is obtained by equation (25) as $0 \mathrm{~s}, 1.62 \mathrm{~s}, 3 \mathrm{~s}$, and the covariance matrix $\Sigma$ can be further obtained as 


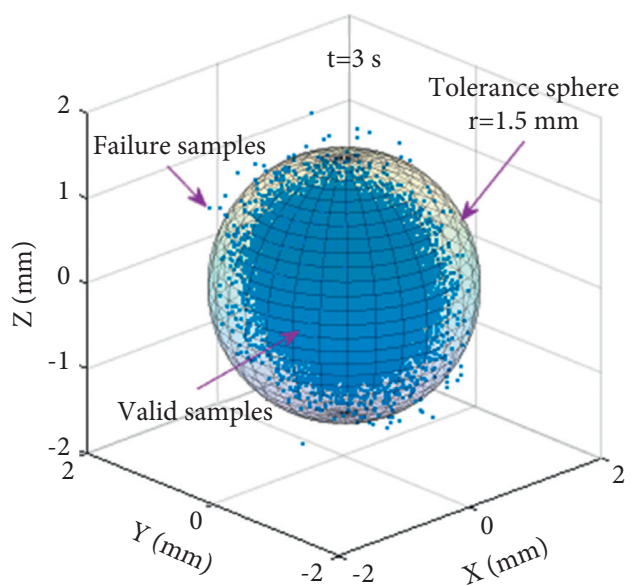

Figure 6: Position of random points.

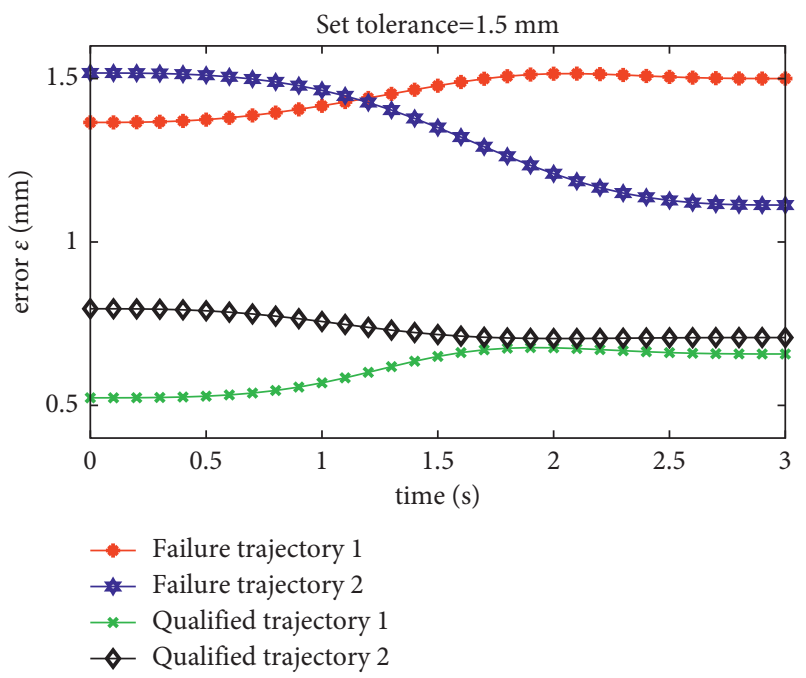

FIgURE 7: Sample trajectories.

$$
\Sigma=\left[\begin{array}{lll}
0.1763 & 0.1729 & 0.1562 \\
0.1729 & 0.1881 & 0.1673 \\
0.1562 & 0.1673 & 0.1621
\end{array}\right] \times 10^{-6}
$$

which is positive definite.

Therefore, reliability at different errors in the $y$ direction can be calculated by equation (36). If $\varepsilon=0.8 \mathrm{~mm}$, then

$$
\begin{aligned}
p f & =1-\int_{-0.8}^{0.8} \frac{1}{(2 \pi)^{r / 2}\left|\Sigma_{\mathbf{y}}\right|} \exp \left[-\frac{1}{2}\left(\mathbf{x}-\boldsymbol{\mu}_{y}\right) \Sigma\left(\mathbf{x}-\boldsymbol{\mu}_{\mathrm{y}}\right)^{T}\right] \mathrm{d} x \\
& =8.40 \times 10^{-2},
\end{aligned}
$$

where $\mu=\left[\begin{array}{lll}0 & 0 & 0\end{array}\right]^{T}$.

The specific values at the error of $0.6 \mathrm{~mm}, 0.8 \mathrm{~mm}$, and $0.1 \mathrm{~mm}$ are counted in Table 3. When the error is $0.6 \mathrm{~mm}$ in $x$-, $y$-, and $z$-directions, the relative errors of the envelope method are $5.8 \%, 4.1 \%$, and $2.9 \%$ compared with the MCS method, and the counterparts of the EEV method are $43.8 \%$, $24.9 \%$, and $46.4 \%$, respectively.
Figure 9 provides the difference in the failure rate of the robot end-effector between the envelope method and the MCS method. As the position error increases, the difference between them becomes smaller, with 90 percent of the data concentrated in the $-1 \times 10^{-2} \sim 0$ region. In conjunction with the preceding analysis, the envelope method is adequate for the kinematic reliability of the redundant robot in a specific direction.

Three algorithms are again introduced to compare their characteristics for the kinematic reliability of the attitude angles, as shown in Figure 10. The envelope method produces results that are slightly closer to those of the MCS method than to those of the EEV method. The reason for this phenomenon is that the deviation of a specific point, which has a large impact on the accuracy of the trajectory of attitude angles, is much larger than the deviation of other points on the trajectory.

According to the data in Table 4, which contains failure rate values for various errors, the envelope method is still superior to the EEV method. In addition, the error of the attitude angle $\beta$ can be ignored, which is too small relative to the other two angles. When the error is $0.015^{\circ}$, the failure rates of the three attitude angles obtained by the envelope method are $4.4 \%(\alpha)$ and $5.5 \%(\gamma)$ relative to the MCS method, better than the result of the EEV. This further shows that the envelope method has high robustness.

On the other hand, we note that the EEV method performs better when calculating the reliability of the attitude angle rather than the position in light of Figures 8 and 10. The summary is important for two reasons: (1) when computing kinematic reliability of attitude angles, the correlation between points at various times is higher, resulting in the accuracy of a single point being similar to the total accuracy. (2) The error in attitude angles of the trajectory is highly volatile, with a certain point having a significant impact on the trajectory.

Figure 11 shows the difference in the failure rate of attitude angles between the envelope method and the MCS method from $0.015^{\circ}$ to $0.042^{\circ}$. The curves in the figure show that the difference between the envelope method and the MCS method is less than $1.5 \times 10^{-2}$ and approaches 0 with the increment of error. This also proves that the envelope method has stronger stability and a wider range of applications.

Another key factor to consider when deciding whether or not a method may be implemented is its efficiency. The envelope method requires $5502 \mathrm{~s}$ to calculate, which is slower than the $2038 \mathrm{~s}$ from the EEV method but much faster than the $36050 \mathrm{~s}$ simulated by the MCS method. Although the calculation efficiency of the envelope method is not as good as that of the EEV method, its accuracy is higher. Therefore, it is more appropriate to use the envelope method to evaluate the kinematic reliability of the robot while taking into account the computation efficiency and accuracy. Taking advantage of the enveloped method, the tolerances of $x$-, $y$-, and $z$-directions are $2 \mathrm{~mm}, 2.3 \mathrm{~mm}$, and $2 \mathrm{~mm}$, respectively. In the same way, the tolerances of $\alpha-, \beta$-, and $\gamma$-attitude angles can be obtained as $0.063^{\circ}, 0.0012^{\circ}$, and $0.054^{\circ}$, respectively. 


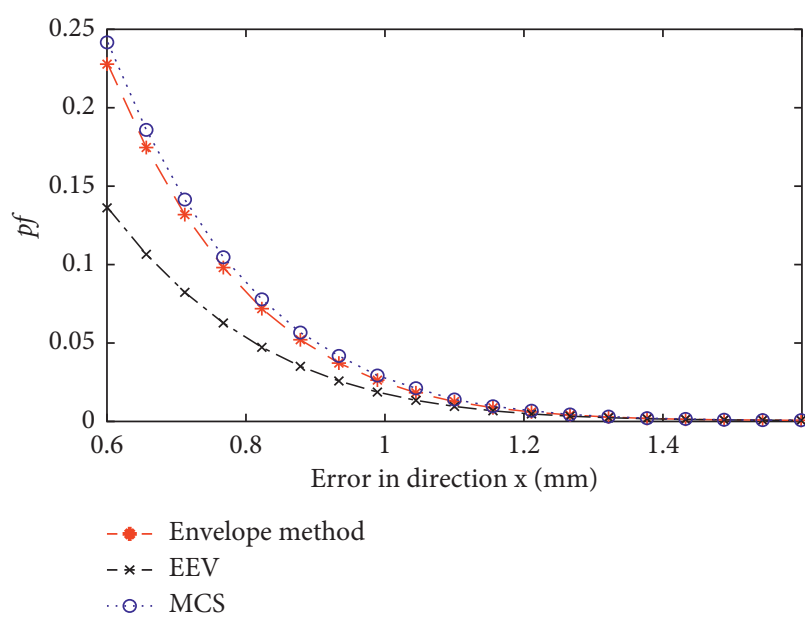

(a)

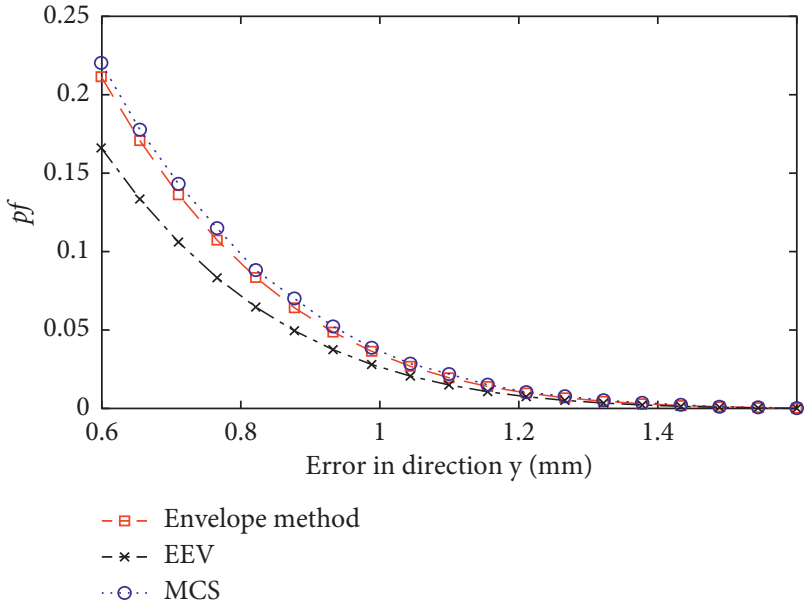

(b)

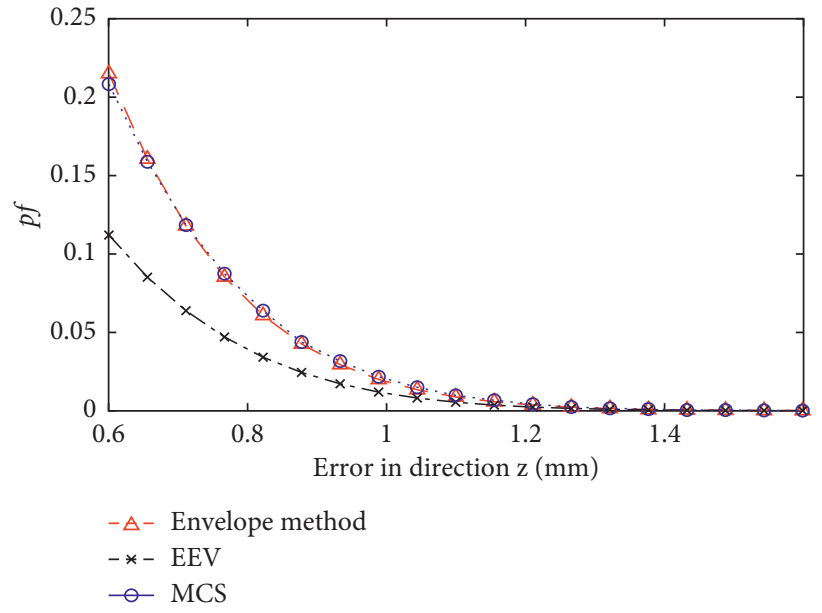

(c)

FIGURE 8: Reliability of the robot position. (a) Failure probability in the $x$ direction. (b) Failure probability in the $y$ direction, and (c) Failure probability in the $z$ direction.

TABLE 3: Reliability of the position.

\begin{tabular}{lcccc}
\hline & Error $(\mathrm{mm})$ & Envelope method & EEV & MCS \\
\hline$x$ & 0.6 & $2.28 \times 10^{-1}$ & $1.36 \times 10^{-1}$ & $2.42 \times 10^{-1}$ \\
$x$ & 0.8 & $7.17 \times 10^{-2}$ & $4.70 \times 10^{-2}$ & $7.77 \times 10^{-2}$ \\
$x$ & 1 & $1.79 \times 10^{-2}$ & $1.30 \times 10^{-2}$ & $2.08 \times 10^{-2}$ \\
$y$ & 0.6 & $2.12 \times 10^{-1}$ & $1.66 \times 10^{-1}$ & $2.21 \times 10^{-1}$ \\
$y$ & 0.8 & $8.40 \times 10^{-2}$ & $6.51 \times 10^{-2}$ & $8.87 \times 10^{-2}$ \\
$y$ & 1 & $2.74 \times 10^{-2}$ & $1.12 \times 10^{-1}$ & $2.91 \times 10^{-2}$ \\
$z$ & 0.6 & $2.15 \times 10^{-1}$ & $3.42 \times 10^{-2}$ & $2.09 \times 10^{-1}$ \\
$z$ & 0.8 & $6.09 \times 10^{-2}$ & $8.12 \times 10^{-3}$ & $6.39 \times 10^{-1}$ \\
$z$ & 1 & $1.35 \times 10^{-2}$ & & $1.47 \times 10^{-2}$ \\
\hline
\end{tabular}

The comprehensive error function of the robot does not usually follow a normal distribution, which is hard to analyze directly through the envelop method. As a result, in this article, the time expansion points in three directions reapproximated as those of the comprehensive error function since the error of three attitude angles is smaller to be ignored based on Tables 3 and 4 . The reliability data collected are depicted in Figure 12, which is calculated approximately by I-PCM (Improved Product of Conditional Marginal) [34] for multidimensional $(n \geq 4)$ normal integral. When the error is $0.6 \sim 1.5 \mathrm{~mm}$, the error of the envelope method relative to the MCS method is $7 \% \sim 68 \%$, which is far inferior to $46 \% \sim 76 \%$ yielded by the EEV method, indicating the envelope method has better performance. Although the calculated result of the envelope method may not reach the ideal state, it obtains a more accurate value close to MCS, 


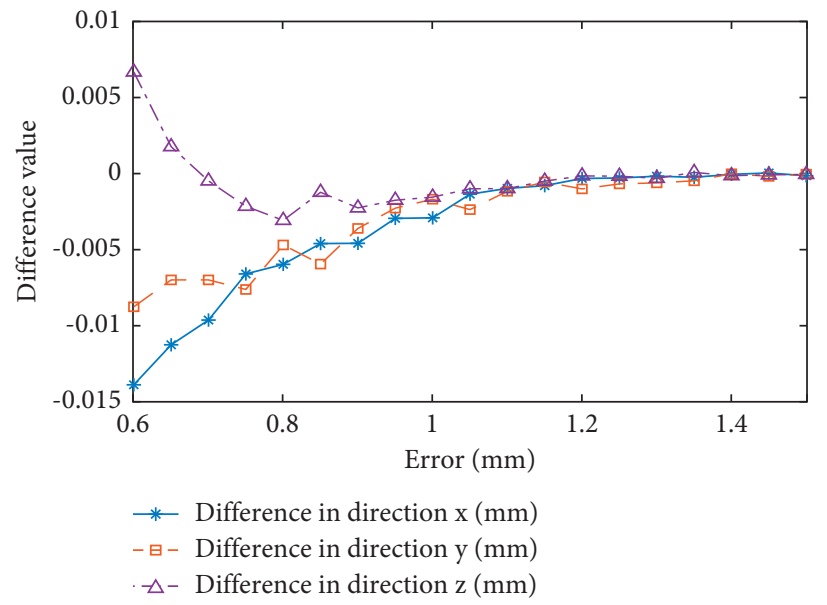

FIGURE 9: Differences between the envelope method and MCS method in directions.
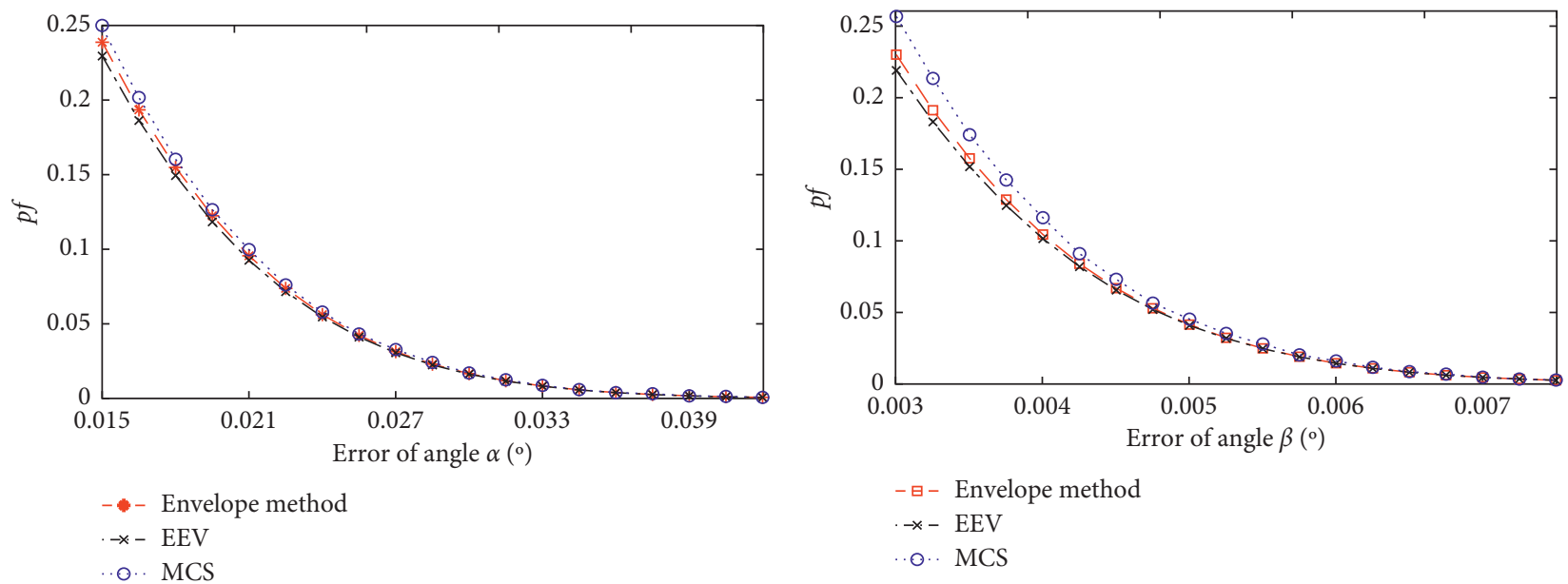

(a)

(b)

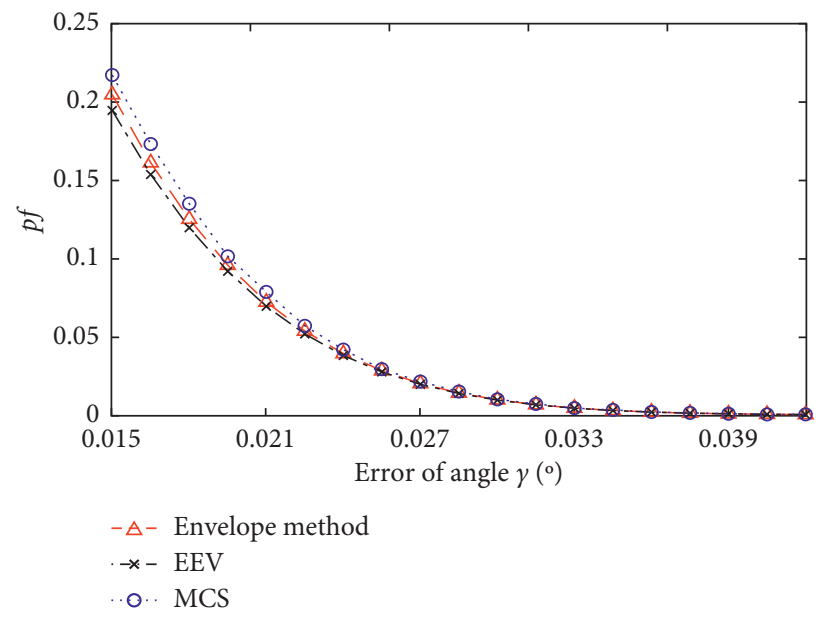

(c)

FIGURE 10: Reliability of robot attitude. (a) Failure probability of angle $\alpha$. (b) Failure probability of angle $\beta$. (c) Failure probability of angle $\gamma$. 
TABLE 4: Reliability of attitude.

\begin{tabular}{lcccc}
\hline & Error (mm) & Envelope method & EEV & MCS \\
\hline$\alpha$ & 0.015 & $2.39 \times 10^{-1}$ & $2.30 \times 10^{-1}$ & $2.50 \times 10^{-1}$ \\
$\alpha$ & 0.021 & $9.58 \times 10^{-2}$ & $9.28 \times 10^{-1}$ & $1.00 \times 10^{-1}$ \\
$\alpha$ & 0.027 & $3.15 \times 10^{-2}$ & $3.07 \times 10^{-2}$ & $3.29 \times 10^{-2}$ \\
$\beta$ & 0.003 & $2.30 \times 10^{-2}$ & $2.19 \times 10^{-2}$ & $2.57 \times 10^{-2}$ \\
$\beta$ & 0.004 & $1.04 \times 10^{-1}$ & $1.01 \times 10^{-1}$ & $1.16 \times 10^{-2}$ \\
$\beta$ & 0.005 & $4.11 \times 10^{-1}$ & $4.05 \times 10^{-2}$ & $4.41 \times 10^{-4}$ \\
$\gamma$ & 0.015 & $2.05 \times 10^{-1}$ & $1.95 \times 10^{-1}$ & $2.17 \times 10^{-1}$ \\
$\gamma$ & 0.021 & $7.23 \times 10^{-2}$ & $6.96 \times 10^{-1}$ & $7.87 \times 10^{-1}$ \\
$\gamma$ & 0.027 & $2.02 \times 10^{-2}$ & $1.96 \times 10^{-2}$ & $2.14 \times 10^{-2}$ \\
\hline
\end{tabular}

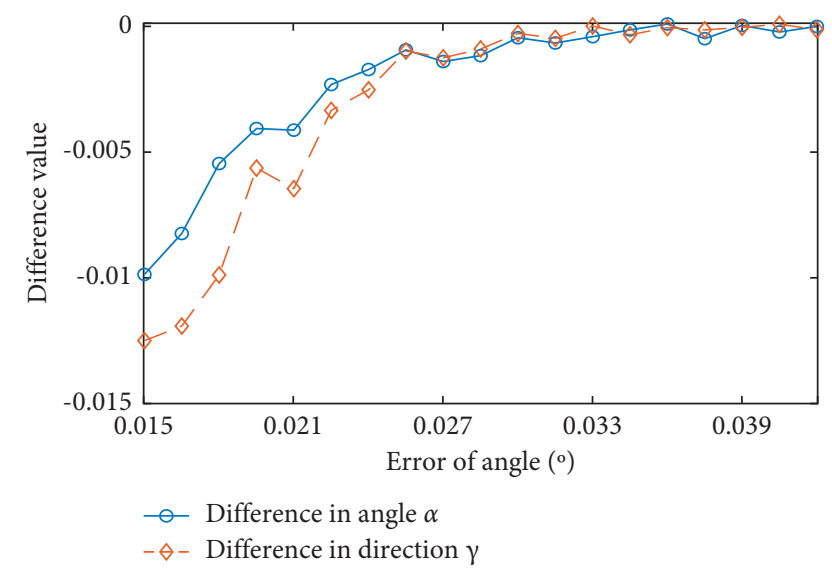

Figure 11: Differences between the envelope method and MCS method in angles.

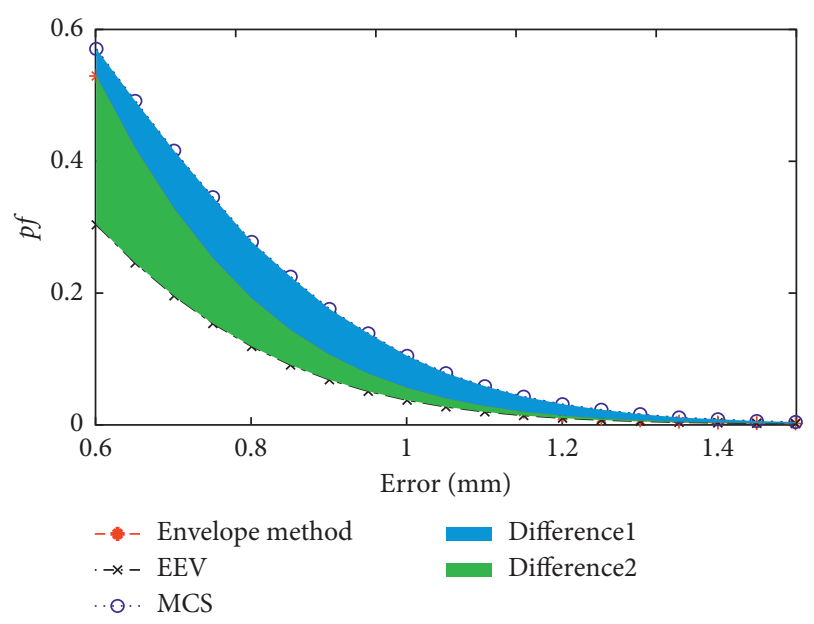

FIGURE 12: Comprehensive reliability of the robot.

and the maximum difference between them is $9.3 \times 10^{-2}$. On the whole, it still proves to be a good solution for the kinematic reliability of the robot.

\section{Conclusion}

The kinematic reliability of a robot is an important indicator for evaluating its performance, and it is inextricably linked to product quality. Although the MCS method is commonly applied to reliability analysis, it often comes with a huge computational cost, which is unbearable in reality. For a 7 DOF redundant robot, this work constructs the error model of the robot trajectory using probabilistic methods and determines the kinematic reliability of the trajectory using the envelope method. Then, the reliability value can be obtained by solving the equation and calculating the multidimensional normal integral, instead of a large number of stochastic simulations.

The simulation example shows that the envelope method is more accurate than the EEV method and closer to the MSC method, with relative errors between the envelope method and MCS of less than 6\% in all orientations and attitude angles. Furthermore, the difference between the envelope method and MCS is no more than $1.5 \times 10^{-2}$, implying that the envelope method is precise. On the other hand, in terms of efficiency, the envelope method, EEV method, and MCS method take $5502 \mathrm{~s}, 2038 \mathrm{~s}$, and $36050 \mathrm{~s}$, respectively, to calculate kinematic reliability. Considering the synthesizing capacity of the solution accuracy and calculation efficiency, the envelope method is the best method for evaluating the kinematic reliability of the robot.

Furthermore, we do not demonstrate whether this method can be used to calculate the reliability of other error models with nonnormal distributions. In the future, we plan to apply this method to implement other tasks, such as spraying and assembly performed by various robots, in order to fully exploit the potential of the proposed method.

\section{Data Availability}

The parameters of the aerial robot we used have been shown in the article. The data of simulation and experiments can be provided if necessary.

\section{Conflicts of Interest}

The authors declare that they have no conflicts of interest.

\section{Authors' Contributions}

Jiahui Gu, Ziyi Li, Shaopeng Kang, and Rui Ma contributed equally to this work.

\section{Acknowledgments}

This work was partially supported by the National Natural Science Foundation of China (52005231), Social Development Science and Technology Support Project of Changzhou (Grant nos. CE20215050 and CE20209002), and Natural Science Research Foundation of Jiangsu Universities (Grant no. 20KJB580005).

\section{References}

[1] W. Xu, L. Han, X. Wang, H. Yuan, and B. Liang, "Intelligent modularized reconfigurable mechanisms for robots: development and experiment," Chinese Journal of Mechanical Engineering = Ji Xie Gong Cheng Xue Bao, vol. 33, no. 1, 2020. 
[2] A. Zakhama, L. Charrabi, and K. Jelassi, "Intelligent selective compliance articulated robot arm robot with object recognition in a multi-agent manufacturing system," International Journal of Advanced Robotic Systems, vol. 16, no. 2, Article ID 1729881419841145, 2019.

[3] Z. Wang, Z. Zhen, Z. Lin, T. Wen, C. Guo, and H. Che, "An adaptive industrial robot spraying planning and control system," in Proceedings of the 46th Annual Conference of the IEEE Industrial Electronics Society (IECON 2020), pp. 47394743, IEEE, Singapore, October 2020.

[4] X. Luo and L. Xu, "On research progress and trend of motion control in industrial robot applications," in Proceedings of the 2020 Chinese Automation Congress (CAC), pp. 5944-5947, IEEE, Shanghai, China, November 2020.

[5] T. Chen, J. Lin, D. Wu, and H. Wu, "Research of calibration method for industrial robot based on error model of position," Applied Sciences, vol. 11, no. 3, p. 1287, 2021.

[6] S. Dereli and R. Köker, "Calculation of the inverse kinematics solution of the 7-DOF redundant robot manipulator by the firefly algorithm and statistical analysis of the results in terms of speed and accuracy," Inverse Problems in Science and Engineering, vol. 28, no. 5, pp. 601-613, 2020.

[7] D. Zhang, Z. Han, F. Wang, and X. Han, "Proficiency of statistical moment-based methods for analysis of positional accuracy reliability of industrial robots," International Journal of Mechanics and Materials in Design, vol. 17, no. 2, pp. 403-418, 2021.

[8] Z. Jiang, M. Huang, X. Tang, and Y. Guo, "A new calibration method for joint-dependent geometric errors of industrial robot based on multiple identification spaces," Robotics and ComputerIntegrated Manufacturing, vol. 71, Article ID 102175, 2021.

[9] Y. Zou and R. Lan, "An end-to-end calibration method for welding robot laser vision systems with deep reinforcement learning," IEEE Transactions on Instrumentation and Measurement, vol. 69, no. 7, pp. 4270-4280, 2019.

[10] H.-M. Qian, Y.-F. Li, and H.-Z. Huang, "Time-variant reliability analysis for industrial robot RV reducer under multiple failure modes using Kriging model," Reliability Engineering \& System Safety, vol. 199, Article ID 106936, 2020.

[11] D. Zhang, Z. Peng, G. Ning, and X. Han, "Positioning accuracy reliability of industrial robots through probability and evidence theories," Journal of Mechanical Design, vol. 143, no. 1, Article ID 011704, 2021.

[12] D. Zhou, E. Pan, X. Zhang, and Y. Zhang, "Dynamic modelbased saddle-point approximation for reliability and reliability-based sensitivity analysis," Reliability Engineering \& System Safety, vol. 201, Article ID 106972, 2020.

[13] T. Fang, C. Jiang, Z. Huang, X. Wei, and X. Han, “Timevariant reliability-based design optimization using an equivalent most probable point," IEEE Transactions on Reliability, vol. 68, no. 1, pp. 175-186, 2018.

[14] J. Kim, W.-J. Song, and B.-S. Kang, "Stochastic approach to kinematic reliability of open-loop mechanism with dimensional tolerance," Applied Mathematical Modelling, vol. 34, no. 5, pp. 1225-1237, 2010.

[15] C.-y. Zhang and G.-c. Bai, "Extremum response surface method of reliability analysis on two-link flexible robot manipulator," Journal of Central South University, vol. 19, no. 1, pp. 101-107, 2012.

[16] W. Wang, H. Gao, C. Zhou, and Z. Zhang, "Reliability analysis of motion mechanism under three types of hybrid uncertainties," Mechanism and Machine Theory, vol. 121, pp. 769-784, 2018.

[17] D. Zhang and X. Han, "Kinematic reliability analysis of robotic manipulator," Journal of Mechanical Design, vol. 142, no. 4, 2020.
[18] J. Wu, D. Zhang, J. Liu, X. Jia, and X. Han, “A computational framework of kinematic accuracy reliability analysis for industrial robots," Applied Mathematical Modelling, vol. 82, pp. 189-216, 2020.

[19] J. Wu, D. Zhang, J. Liu, and X. Han, "A moment approach to positioning accuracy reliability analysis for industrial robots," IEEE Transactions on Reliability, vol. 69, no. 2, pp. 699-714, 2019.

[20] Q. Zhao, J. Guo, and J. Hong, "System kinematic reliability analysis for robotic manipulators under rectangular and spherical tolerant boundaries," Journal of Mechanisms and Robotics, vol. 13, no. 1, Article ID 011004, 2021.

[21] J. Zhang, J. Wang, and X. Du, "Time-dependent probabilistic synthesis for function generator mechanisms," Mechanism and Machine Theory, vol. 46, no. 9, pp. 1236-1250, 2011.

[22] G. J. Savage, X. Zhang, Y. K. Son, and M. D. Pandey, "Reliability of mechanisms with periodic random modal frequencies using an extreme value-based approach," Reliability Engineering \& System Safety, vol. 150, pp. 65-77, 2016.

[23] Z. Wang, Z. Wang, S. Yu, and K. Zhang, "Time-dependent mechanism reliability analysis based on envelope function and vine-copula function," Mechanism and Machine Theory, vol. 134, pp. 667-684, 2019.

[24] Q. Zhao, J. Guo, D. Zhao, D. Yu, and J. Hong, "Time-dependent system kinematic reliability analysis for robotic manipulators," Journal of Mechanical Design, vol. 143, no. 4, Article ID 041704, 2021.

[25] M. D. Pandey and X. Zhang, "System reliability analysis of the robotic manipulator with random joint clearances," Mechanism and Machine Theory, vol. 58, pp. 137-152, 2012.

[26] H. L. Vieira, A. T. Beck, and M. M. da Silva, "Combined interval analysis-Monte Carlo simulation approach for the analysis of uncertainties in parallel manipulators," Meccanica, pp. 1-15, 2021.

[27] P. I. Corke, "A simple and systematic approach to assigning denavit-hartenberg parameters," IEEE Transactions on Robotics, vol. 23, no. 3, pp. 590-594, 2007.

[28] P. Huang, H.-Z. Huang, Y.-F. Li, and H. Li, "Positioning accuracy reliability analysis of industrial robots based on differential kinematics and saddlepoint approximation," Mechanism and Machine Theory, vol. 162, Article ID 104367, 2021.

[29] A. Fernandez and D. Baleanu, "The mean value theorem and Taylor's theorem for fractional derivatives with Mittag-Leffler kernel," Advances in Difference Equations, vol. 2018, no. 1, pp. 1-11, 2018.

[30] X. P. Du, "Time-dependent mechanism reliability analysis with envelope functions and first-order approximation," Journal of Mechanical Design, vol. 136, no. 8, pp. 1-7, 2014.

[31] T. Jin, B. Janamanchi, and Q. Feng, "Reliability deployment in distributed manufacturing chains via closed-loop six sigma methodology," International Journal of Production Economics, vol. 130, no. 1, pp. 96-103, 2011.

[32] L.-C. T. Wang and C. C. Chen, "A combined optimization method for solving the inverse kinematics problems of mechanical manipulators," IEEE Transactions on Robotics and Automation, vol. 7, no. 4, pp. 489-499, 1991.

[33] J. Li, J.-b. Chen, and W.-l. Fan, "The equivalent extreme-value event and evaluation of the structural system reliability," Structural Safety, vol. 29, no. 2, pp. 112-131, 2007.

[34] X.-X. Yuan and M. D. Pandey, "Analysis of approximations for multinormal integration in system reliability computation," Structural Safety, vol. 28, no. 4, pp. 361-377, 2006. 